\title{
"STAGGERING" EFFECT IN EVEN-EVEN NUCLEI WITH QUADRUPOLE AND OCTUPOLE DEFORMATIONS
}

\author{
M.S. NADIRBEKOV, G.A. YULDASHEVA \\ Institute of Nuclear Physics, Academy of Sciences of Republic Uzbekistan \\ (Ulugbek, Tashkent 100214, Uzbekistan)
}

PACS 21.10.-k

(C) 2011

The $\Delta I=1$ "staggering" effect is investigated in the energy spectrum of positive and negative parities of the yrast band of eveneven actinide nuclei. The "staggering" effect is described by the formula proportional to the discrete approximation of the fourthorder derivative of the function $\Delta E(I)$. It is shown that the "staggering" effect is characterized by the "zigzag" behavior and does not vanish within the observed range of angular momenta.

\section{Introduction}

Rotational nuclear spectra were previously determined by quadrupole deformations $\left(\beta_{2}\right)$ [1] corresponding to nuclei in the form of an ellipsoid of revolution. In this case, low-lying excited states of even-even nuclei are described in the geometrical approximation as levels corresponding to harmonic oscillations and rotations of a deformed surface [1].

Octupole deformations $\left(\beta_{3}\right)$ take place in actinides [2] and in nuclei with the mass number $A \approx 150[3,4]$ corresponding to pearlike nuclear shapes [5-7].

The properties of nuclei with octupole degrees of freedom have been recently widely studied in the framework of various geometrical, algebraic, and microscopic approximations when describing the nuclear structure [7]. It is worth noting that quadrupole and octupole deformations of the nuclear surface are difficult to be simultaneously taken into account both in the microscopic approximation, due to the violation of the reflection symmetry of a nuclear shape, and in the geometrical one, due to the complicacy of the determination of the total tensor of inertia of the system. However, one can consider a simplified form of this problem for axially symmetric nuclei, i.e. at $K=0$ ( $K$ is the projection of the total angular momentum $I$ on the symmetry axis of a nucleus) in the geometrical approximation.

The energy levels of positive- and negative-parity excited states, as well as the reduced probabilities of dipole, quadrupole, and octupole transitions between these states, were determined in [8] for the case of the axially symmetric nuclei ${ }^{144,146} \mathrm{Ba},{ }^{146} \mathrm{Ce}$, ${ }_{146,148,150} \mathrm{Nd},{ }^{150} \mathrm{Sm},{ }^{151} \mathrm{Pm},{ }^{220,222} \mathrm{Rn},{ }^{217,219,221} \mathrm{Fr}$, ${ }^{218-228} \mathrm{Ra},{ }^{219,223,225,227} \mathrm{Ac}$ and ${ }^{220-229} \mathrm{Th}$, with the use of the Davidson potential [9] for surface deformations.

The alternative parity energy levels of the axially symmetric even-even nuclei ${ }^{150} \mathrm{Sm},{ }^{154} \mathrm{Dy},{ }^{162} \mathrm{Dy},{ }^{232} \mathrm{Th}$, and $232,236,238 \mathrm{U}$ were also considered in [10] for the oscillatory potential energy of surface deformations. The energy spectrum and the wave functions of excited states were obtained. The energy levels are described by three theoretical parameters: the energy multiplier $\hbar \omega$, the parameter $\varepsilon_{\nu}^{\prime}$ proportional to the eigenvalue of the angular part of the Schrödinger equation in polar coordinates $\varepsilon_{\nu}$, and the dimensionless parameter $\mu$ that describes the "softness" of a nucleus with respect to a deformation of its surface.

In the cited works $[8,10]$, nuclei with octupole deformation have two minima of the potential energy corresponding to the opposite values of the octupole deformation parameter $\beta_{3}$. They are also characterized by the two-fold level degeneracy which is eliminated due to a tunnel transition through the potential barrier separating the $\beta_{3}$ and $-\beta_{3}$ configurations that allow one to simultaneously reproduce bands with positive and negative parities. These bands are related to symmetric and antisymmetric combinations of the wave functions [8]. The parameters of the quadrupole $\beta_{2}$ and octupole $\beta_{3}$ deformations in these nuclei are of one order of magnitude [11]. 
The excited-state energies were described in $[8,10]$ with the use of the following potential energies of surface deformations: Davidson potential [9] and oscillatory one [12]. These potentials are widely used to describe the energy levels of individual bands of excited states of different multipolarities [13-15].

Another difference between the cited works concerns the kinetic part of the Hamiltonian. In [8], it corresponds to the Hamiltonian for nonaxial even-even nuclei [12]

$$
\begin{aligned}
& \hat{T}_{1}=-\sum_{\lambda=2,3} \frac{\hbar^{2}}{2 B_{\lambda}} \frac{1}{\beta_{\lambda}^{3}} \frac{\partial}{\partial \beta_{\lambda}}\left(\beta_{\lambda}^{3} \frac{\partial}{\partial \beta_{\lambda}}\right)= \\
& =-\frac{\hbar^{2}}{2 B_{2}}\left\{\frac{3}{\beta_{2}} \frac{\partial}{\partial \beta_{2}}+\frac{\partial^{2}}{\partial \beta_{2}^{2}}\right\}-\frac{\hbar^{2}}{2 B_{3}}\left\{\frac{3}{\beta_{3}} \frac{\partial}{\partial \beta_{3}}+\frac{\partial^{2}}{\partial \beta_{3}^{2}}\right\},
\end{aligned}
$$

where $B_{2}$ and $B_{3}$ are the mass parameters.

The kinetic part of the Hamiltonian in [10] corresponds to the Hamiltonian of axially symmetric nuclei $[16]$

$$
\begin{aligned}
& \hat{T}_{2}=-\sum_{\lambda=2,3} \frac{\hbar^{2}}{2 B_{\lambda}} \frac{1}{\beta_{\lambda}^{2}} \frac{\partial}{\partial \beta_{\lambda}}\left(\beta_{\lambda}^{2} \frac{\partial}{\partial \beta_{\lambda}}\right)= \\
& =-\frac{\hbar^{2}}{2 B_{2}}\left\{\frac{2}{\beta_{2}} \frac{\partial}{\partial \beta_{2}}+\frac{\partial^{2}}{\partial \beta_{2}^{2}}\right\}-\frac{\hbar^{2}}{2 B_{3}}\left\{\frac{2}{\beta_{3}} \frac{\partial}{\partial \beta_{3}}+\frac{\partial^{2}}{\partial \beta_{3}^{2}}\right\} .
\end{aligned}
$$

Calculating the difference between these Hamiltonians, we obtain

$\hat{T}_{1}-\hat{T}_{2}=\frac{\hbar^{2}}{2 B_{2}} \frac{1}{\beta_{2}} \frac{\partial}{\partial \beta_{2}}-\frac{\hbar^{2}}{2 B_{3}} \frac{1}{\beta_{3}} \frac{\partial}{\partial \beta_{3}}$.

After passing to the polar coordinates, this difference vanishes $[8,10]$, and there remains only the second term of formula (9) present in [8] and absent in [10]. This term was omitted in the further computations due to its small contribution to the energies of levels at large spins $[13,14]$. Thus, the difference of the kinetic parts of Hamiltonians (1) and (2) does not make any contribution to the final result.

There exist several well-studied types of deviations of the nuclear collective motion (in the first approximation) from the purely rotational one $[1,17]$. These deviations result in high-order effects in the structure of the nuclear rotational spectrum, such as "squeezing", "backbending", and "staggering" [18-22]. Investigations of these fine effects in the structure of collective interaction and the corresponding energy spectra of nuclei suppose a complex behavior of collective characteristics. Among them, there are rotation, vibration, pair vibration, and others $[1,12,13]$. These collective modes represent complex and diverse excitations with simultaneous participation of many nucleons that, nevertheless, can be theoretically described with the use of a small number of degrees of freedom $[1,12,13]$.

The application of discrete approximations of highorder derivatives of the given nuclear characteristic as a function of a particular physical quantity demonstrates various kinds of "staggering" effects that bear information about fine properties of the nuclear interaction and corresponding high-order correlations in the collective dynamics of a system.

The "staggering" effects represent the branchings of rotational bands in the sequence of states differing by several units of the angular momentum. These $\Delta I=1$, $\Delta I=2$, and $\Delta I=4$ "staggering" effects are observed in the energy bands of superdeformed nuclei [21-24]. These effects are very well studied in even-even nuclei [1] and allow one to test various collective models [25].

The $\Delta I=2$ "staggering" effect appears in the case where levels with $I=2,6,10,14, \ldots$ are shifted with respect to those with $I=0,4,8,12, \ldots$, i.e. the level with the angular momentum $I$ is shifted relative to its neighboring levels with the angular momentum $I \pm 2$ in the energy levels of the principal band of deformed nuclei [24].

It was already mentioned that, in the geometrical model, the appearance of reflection-asymmetric shapes in atomic nuclei is related to the manifestation of the octupole degree of freedom. The main physical characteristic of a system with the reflection asymmetry is related to the violation of the $R$ and $P$ symmetries. It is known that these symmetries are violated separately, whereas the system remains invariant with respect to their product $P R^{-1}$ [1]. In this case, the spectrum of the system is characterized by the presence of energy bands with angular momenta of alternative parity. Consequently, a band of negative parity with the sequence of levels $I^{\pi}=1^{-}, 3^{-}, 5^{-}, 7^{-}, \ldots$, combining with a band of positive parity with the sequence of levels $I^{\pi}=0^{+}, 2^{+}, 4^{+}, 6^{+}, \ldots$, forms a band with the sequence of levels $I^{\pi}=0^{+}, 1^{-}, 2^{+}, 3^{-}, 4^{+}, 5^{-}, \ldots$.

Such a band is observed in even-even rare-earth nuclei and in actinides $[2-4,6,7,26,27]$. In these bands of even-even nuclei, the energy levels with odd $I$ and negative parity are shifted with respect to the energy levels with even $I$ and positive parity. In other words, the 

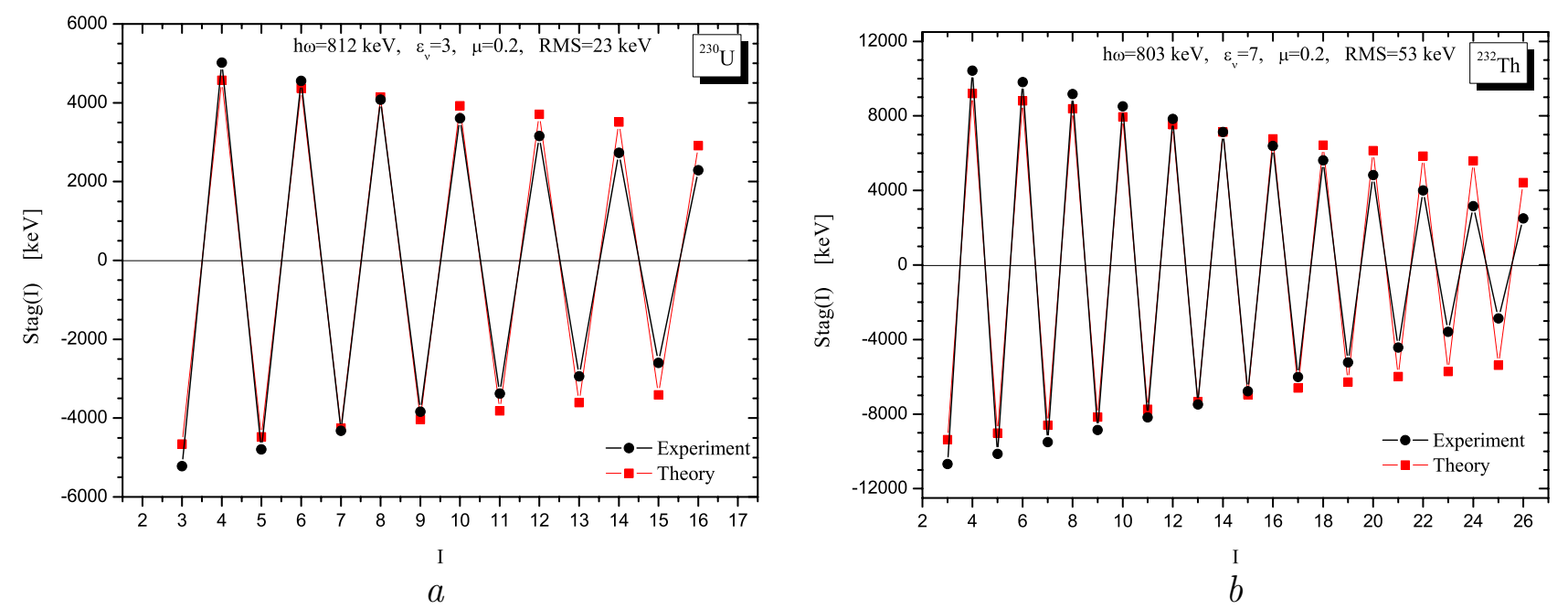

Fig. 1. Theoretical and experimental [33] behaviors of the "staggering" effect in the energy spectra of the $\mathrm{U}^{230}$ and Th ${ }^{232}$ nuclei

level with angular momentum $I$ is shifted relative to the neighboring state with the angular momentum $I \pm 1$ [18]. This quantity, usually called the odd-even "staggering" or $\Delta I= \pm 1$ "staggering", must vanish if even and odd energy levels form a single band.

The $\Delta I=1$ "staggering" effect of such a kind takes place in the $\gamma$-band of even-even nuclei, but it differs from the "staggering" effect of the energy bands of octupole-deformation nuclei in that the energy levels in the $\gamma$-band of these nuclei have a positive parity alone [25].

In [28], the authors discussed the experimental behavior of the odd-even "staggering" effect in the bands of the rare-earth nuclei ${ }^{144,146} \mathrm{Ba},{ }^{150,152,154} \mathrm{Sm},{ }^{154,156,162} \mathrm{Dy}$, ${ }^{152,154,156,160} \mathrm{Gd}$, and ${ }^{162,164} \mathrm{Er}$, as well as the actinides ${ }^{220,224} \mathrm{Ra},{ }^{226,228,230,232,234} \mathrm{Th}$, and ${ }^{230,232,234,236,238} \mathrm{U}$.

These effects have different forms but their behavior is the same. It is noted that the energy bands of the above-mentioned nuclei are free from the "backbending/upbending" effects [29].

It is worth noting that the odd-even "staggering" effect with the angular momentum $I \pm 1$ was not considered in $[8,10]$. The analysis and the interpretation of this effect are, however, of special interest, because it bears information about properties of the collective dynamics in various nuclei. In addition, it is very sensitive to the fine structure of the rotational spectrum, and provides an explicit $\Delta I=1$ "staggering" behavior in various rotational bands.

This work considers the behavior of the "staggering" effect in the energy spectra of even-even actinide nuclei.

\section{2. $I \pm 1$ "Staggering" Effect in Axially Symmetric Even-even Nuclei}

Let us consider the odd-even "staggering" effect proportional to the discrete approximation of the fourth-order derivative of the function $\Delta E(I)=E(I+1)-E(I)$ presented by the formula [30]:

$$
\begin{aligned}
& \operatorname{Stag}(I)=6 \Delta E(I)-4 \Delta E(I-1)- \\
& -4 \Delta E(I+1)+\Delta E(I+2)+\Delta E(I-2),
\end{aligned}
$$

where $E(I)$ is the energy of excited states. It is worth noting that there are other alternative formulas for the description of the behavior of the odd-even $\Delta I=1$ "staggering" effect [28]. However, its behavior does not depend on the form of these formulas [28].

It is traditionally believed that the odd-even "staggering" effect in bands with octupole deformation starts from relatively high values at low spins and after that gradually decreases to zero, thus indicating the formation of a reflection-asymmetric band. However, using the recent data obtained for actinides [31], it was found that, in light actinides, the odd-even "staggering" effect demonstrates a "zigzag" behavior [30]. In other words, the quantity measuring the odd-even "staggering" effect does not remain zero after reaching a vanishing value for the first time, but goes on to oscillate (in the absolute magnitude) with increase in $I$, by forming a zigzag shape.

As an example, Figures 1-3 show the theoretical and experimental [33] behaviors of the $\Delta I=1$ "staggering" effect in the energy spectra of ${ }^{232} \mathrm{Th}$ and ${ }^{230,234,236,238} \mathrm{U}$ 

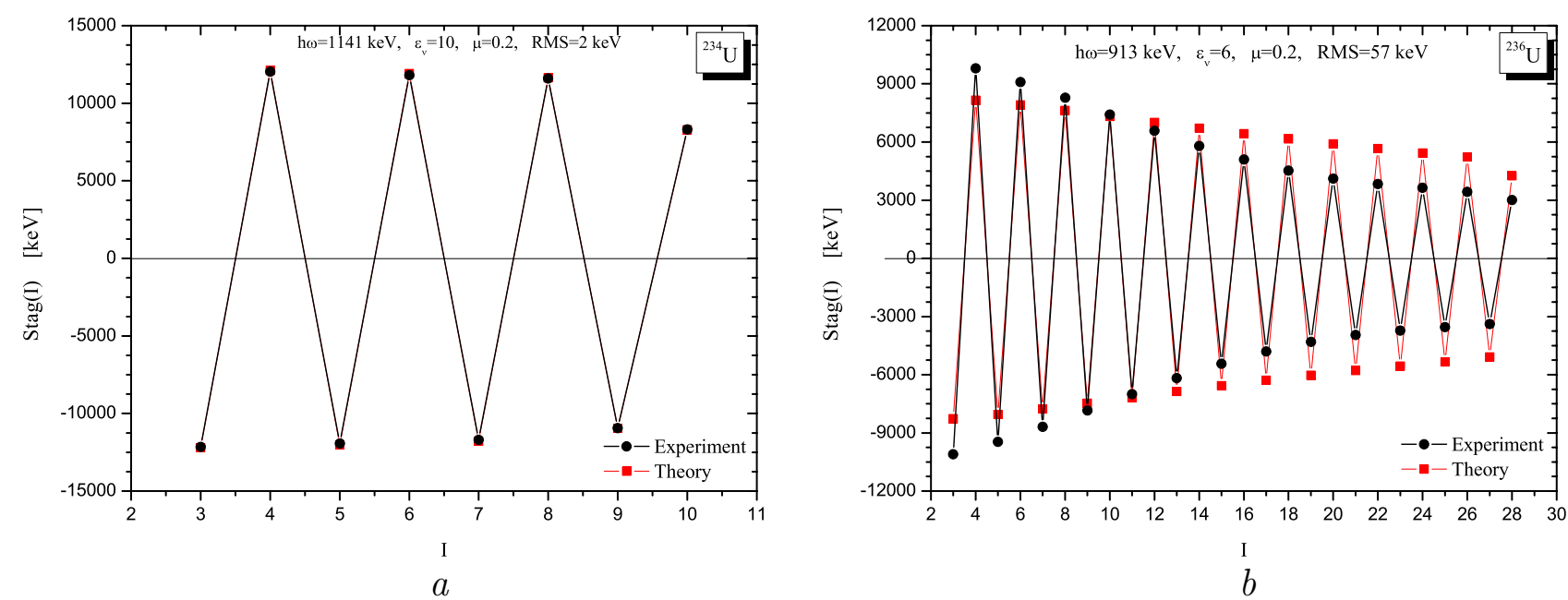

Fig. 2. Theoretical and experimental [33] behaviors of the "staggering" effect in the energy spectra of $\mathrm{U}^{234}$ and $\mathrm{U}^{236}$ nuclei

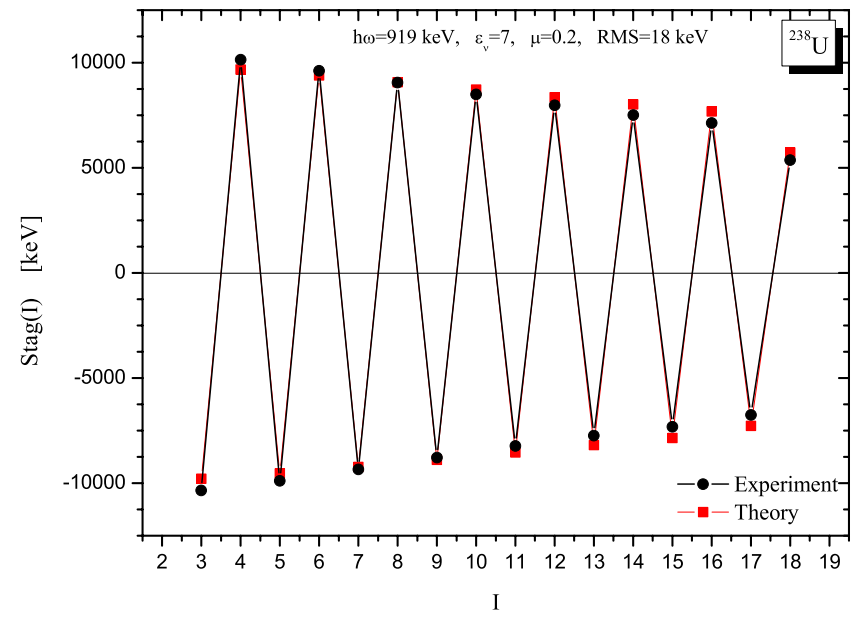

Fig. 3. Theoretical and experimental [33] behaviors of the "staggering" effect in the energy spectra of $U^{238}$ nucleus

nuclei. The figures also demonstrate the values of the parameters used in the proposed model for the description of the energy levels and the root-mean-square deviation of the theoretical and experimental values of these levels that lie within the allowed range. One can see from the figures that the "staggering" effect demonstrates a "zigzag" behavior and does not vanish in the observed range of angular momenta. The theoretical and experimental behaviors of this effect for ${ }^{230,234,238} \mathrm{U}$ nuclei are in good agreement.

The highest value of the "staggering" effect is characteristic of ${ }^{232} \mathrm{Th}$ nucleus at low values of the angular momentum $I$. With increase in $I$, the value of the "stag- gering" effect rapidly decreases, moreover, the experimental values decrease faster than the theoretical ones. A similar picture can be observed in the behavior of the "staggering" effect in ${ }^{236} \mathrm{U}$ nucleus.

It is worth noting that there exists a certain deviation in the behavior of the "staggering" effect in the considered nuclei at large spins, which is related to a deviation of their energy spectrum from the purely rotational one. So, the behavior of the "staggering" effect in ${ }^{230,234,238} \mathrm{U}$ nuclei is similar to that in some rare-earth ones [34].

Consequently, the obtained results allow one to make a conclusion that, at low values of angular momentum of the energy spectrum, the "staggering" effect appears mainly due to the changing parity, whereas it is caused at high angular momenta by the interaction between the rotation of a nucleus as a whole and the deformation of its surface.

Thus, the proposed model describes well the behavior of the "staggering" effect of these nuclei. It also allows one to compare the effects of collective interaction and nuclear shapes in various nuclear regions. It is worth noting that the "backbending/upbending" effects are not registered in the energy levels of the considered nuclei $[32,33]$.

The theoretical values of the energy spectra of the studied nuclei are calculated using formula (31) from [10].

The authors thank Candidate of Phys.-Math. Sci. A.V. Khugaev for his interest to this work and critical remarks. The work is fulfilled under support of Grant 
FA-F2-F-077 of the Academy of Sciences of Republic Uzbekistan.

1. A. Bohr and B.R. Mottelson, Nuclear Structure Vol. II: Nuclear Deformations (World Scientific, Singapore, 1998).

2. P. Schüler et al., Phys. Lett. B 174, 241 (1986).

3. W.R. Phillips et al., Phys. Rev. Lett. 57, 3257 (1986).

4. R.K. Sheline and P.C. Sood, Phys. Rev. C 34, 2362 (1986).

5. G.A. Leander, R.K. Sheline, P. Moller, P. Olanders, I. Ragnarsson, and A.J. Sierk, Nucl. Phys. A 388, 452 (1982); G.A. Leander and R.K. Sheline, Nucl. Phys. A 413, 375 (1984).

6. I. Ahmad and P.A. Butler, Annu. Rev. Nucl. Part. Sci. 43, 71 (1993).

7. P.A. Butler and W. Nazarewicz, Rev. Mod. Phys. 68, 349 (1996).

8. V.Yu. Denisov and A.Ya. Dzyublik, Nucl. Phys. A 589, 17 (1995).

9. J.P. Elliot and J.A. Evans, Phys. Lett. B 169, 309 (1986).

10. Sh. Sharipov, M.S. Nadyrbekov, and G.A. Yuldasheva, Ukr. Fiz. Zh. 53, 112 (2008).

11. P.A. Butler and W. Nazarewicz, Nucl. Phys. A 533, 249 (1991).

12. A.S. Davydov and A.A. Chaban, Nucl. Phys. 20, 499 (1960).

13. A.S. Davydov, Excited States of Atomic Nuclei (Atomizdat, Moscow, 1967) (in Russian).

14. V.K. Tartakovskii, Yad. Fiz. 50, 335 (1989); P.V. Skorobogatov, Yad. Fiz. 15, 220 (1972).

15. V.Yu. Denisov and A.Ya. Dzyublik, Yad. Fiz. 56, 30 (1993).

16. A.G. Sitenko and V.K. Tartakovskii, Lectures on the Theory of the Nucleus (Pergamon Press, Oxford, 1975).

17. P. Ring and P. Shuck, The Nuclear Many-Body Problem (Springer, Heidelberg, 1980).

18. Dennis Bonatsos, C. Daskaloyannis, S.B. Drenska, N. Karoussos, J. Maruani, N. Minkov, P.P. Raychev, and R.P. Roussev, Phys. Rev. A 60, 253 (1999).

19. R.M. Lieder and H. Ryde, Advances in Nuclear Physics, edited by M. Baranger and E. Vogt (Plenum Press, New York, 1978), p. 1.

20. F.S. Stephens and R.S. Simon, Nucl. Phys. A 138, 257 (1972).
21. S. Flibotte et al., Phys. Rev. Lett. 71, 4299 (1993); Nucl. Phys. A 584, 373 (1995).

22. B. Cederwall, et al., Phys. Rev. Lett. 72, 3150 (1994).

23. D. Bonatsos, C. Daskaloyannis, S. Drenska, G. Lalazissis, N. Minkov, P. Raychev, and R. Roussev, Phys. Rev. A 54, R2533 (1996).

24. C.S. Wu and Z.N. Zhou, Phys. Rev. C 56, 1814 (1997); L.A. Wu and H. Toki, Phys. Rev. C 56, 1821 (1997).

25. D. Bonatsos, Phys. Lett. B200, 1 (1988).

26. P.C. Sood, D.M. Headly, and R.K. Sheline, At. Data Nucl. Data Tables 47, 89 (1991); P.C. Sood, D.M. Headly, and R.K. Sheline, At. Data Nucl. Data Tables 51, 273 (1992).

27. W.R. Phillips et al., Phys. Lett. B 212, 402 (1988).

28. Dennis Bonatsos, C. Daskaloyannis, S. B. Drenska, N. Fotiades, N. Minkov, P.P. Raychev, and R.P. Roussev, arXiv:nucl-th/0111003 v1 1 Nov 2001.

29. M.J.A. de Voigt, J. Dudek, and Z. Szymanski, Rev. Mod. Phys. 55, 949 (1983).

30. Dennis Bonatsos, C. Daskaloyannis, S.B. Drenska, N. Karoussos, N. Minkov, P.P. Raychev, and R.P. Roussev, Phys. Rev. C 62, 024301 (2000).

31. J.F.C. Cocks et al., Phys. Rev. Lett. 78, 2920 (1997); J.F.C. Cocks et al., Nucl. Phys. A 645, 61 (1999).

32. I.M. Pavlyuchenkov, Usp. Fiz. Nauk 133, 193 (1981).

33. http://www.nndc.bnl.gov/ensdf/

34. N. Minkov et al., Phys. Rev. C 73, 044315 (2006).

Received 09.11.10.

Received Translated from Russian by H.G. Kalyuzhna

\section{"STAGGERING" ЕФЕКТ У ПАРНО-ПАРНИХ ЯДРАХ 3 КВАДРУПОЛЬНОЮ І ОКТУПОЛЬНОЮ ДЕФОРМАЦІЯМИ}

М.С. Надирбеков, Г.А. Юлдашева

Р ез ю м е

Досліджено $\Delta I=1$ "staggering" ефект в енергетичному спектрі позитивної і негативної парності іраст-полоси парно-парних ядер в області актинідів. Використано формулу "staggering" ефекту, який пропорційний дискретному наближенню похідної четвертого порядку від функції $\Delta E(I)$. Показано, що "staggering" ефект має "зигзагоподібну" поведінку і зникнення цього ефекту в енергетичному спектрі не відбувається в межах спостережуваної області кутового моменту. 